\title{
A novel acoustic emission parameter for predicting rock failure during Brazilian test based on cepstrum analysis
}

\author{
Honglei Wang ${ }^{1,2}$, Zhenlei $\mathrm{Li}^{1,2, *}$, Xueqiu $\mathrm{He}^{1,2}$, Dazhao Song ${ }^{1,2}$, and Haifeng Guo ${ }^{1,2}$ \\ ${ }^{1}$ School of Civil and Resource Engineering, University of Science and Technology Beijing, 100083 \\ Beijing, China \\ ${ }^{2}$ Key Laboratory of Ministry of Education for Efficient Mining and Safety of Metal Mine, University \\ of Science and Technology Beijing, 100083 Beijing, China
}

\begin{abstract}
Acoustic emission (AE) is widely used in the monitoring of coal and rock stability and early warning of dynamic disasters in mines. In this work, the Brazilian split test was carried out on limestone samples along with collecting full waveform $\mathrm{AE}$ signal during the entire loading process. The linear cepstrum coefficient (LCC) was used as the characteristic parameter of $\mathrm{AE}$ to analyze the correlation between the LCC of AE and the load of the samples. The results show that the LCC is an effective AE characteristic parameter. The first to fourth parameters of the LCC calculated using $1 \mathrm{~s} \mathrm{AE}$ waveform meet the linear change relationship with the load of the samples. The correlation coefficients are 0.969, 0.943, 0.925, 0.833 , respectively. The LCC of AE proposed in this work can be used as a characteristic parameter for predicting the tensile failure of rocks, which is helpful to improve the application effect of $\mathrm{AE}$ in the monitoring of coal and rock dynamic disasters and the stability of high and steep slopes.

Keywords. acoustic emission (AE); linear cepstrum coefficient (LCC); dynamic disaster; coal and rock stability.
\end{abstract}

\section{Introduction}

It is generally known that the tensile strength of rock is far less than the compressive strength. The tensile strength plays a vital role in determining important factors such as the load carrying capacity and damage accumulation [1]. Uniaxial tensile strength test is a direct test method of rock tensile strength, but the related sample is complicated and hard to prepare. Therefore, a number of alternate tests are used which infer the tensile strength without making a direct measurement [1]. The Brazilian test is most common because of its simple specimen preparation, experimental procedure and data reduction [2].

Cepstrum based methods were firstly used in the echo detection of seismic signal, and then were applied in the fields of sound signal processing, machine diagnostics and operational modal analysis, etc. The application in these fields has all achieved satisfactory results. Braun noted some unusual aspects of cepstrum based methods that have many appealing properties as a signal processing tool [3]. The cepstrum is a function of quefrency,

\footnotetext{
*Corresponding author: lizhenlei@ustb.edu.cn
} 
that defined as the inverse Fourier transform of the log-spectrum of a stationary random process [4]. Randall et al. [5, 6] reviewed the application status of cepstral coefficient methods in mechanical field.

Among the cepstrum based methods, cepstral coefficient methods are the most prominent ones, which represent audio based on perception of human auditory systems [7]. It not only has excellent performance in the field of speech processing such as speaker recognition [8], music style recognition [9] and language recognition [10], but also has been successfully applied to construction equipment recognition [11], rock burst sound signal recognition [12] and other engineering fields. Mei et al. [13] used cepstrum coefficient and analyzed signals of bridge deformation, midspan stiffness etc. They not only identified damage to the bridge, but also quantitatively analyzed the damage degree.

Cepstrum based method is an effective method to process monitoring signals. However, the application of cepstral coefficient to the analysis of AE signal in Brazilian split test of rock has rarely been reported. In this work, the full waveform data of AE during Brazilian split test of limestone were firstly collected and used to calculate the LCC. The correlation between LCC of AE and rock tensile stress was then analyzed by using linear regression method. The LCC of AE proposed in this work enriches the AE signal analysis, and is helpful to improve the application effect of $\mathrm{AE}$ in the monitoring of coal and rock dynamic disasters and the stability of high and steep slopes.

\section{Methodology}

Power cepstrum has a very long history, since the first paper was published in 1963, two years before the publication of the FFT algorithm [5, 6]. Cepstrum was defined as the inverse Fourier transform of the log-spectrum of a stationary random process [4]. It is also known as quefrency and its dimension is the unit of time. The block diagram of solving power cepstrum is shown in Fig.1(a) [14]. The cepstrum solution process can be divided into 4 steps: Step 1, use fast Fourier transform (FFT) to solve the frequency spectrum of the signal; step 2, solve the absolute value of the spectrum to get the power spectrum of the signal; step 3, calculate the logarithm of the power spectrum; step 4, perform inverse Fourier transform on the logarithm of the power spectrum to obtain the power complex cepstrum of the signal. If the power spectrum is transformed by inverse Fourier transform after step 2, the autocorrelation coefficient of the signal will be obtained.

(a)

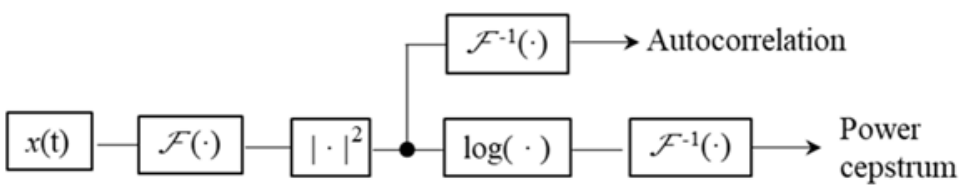

(b)

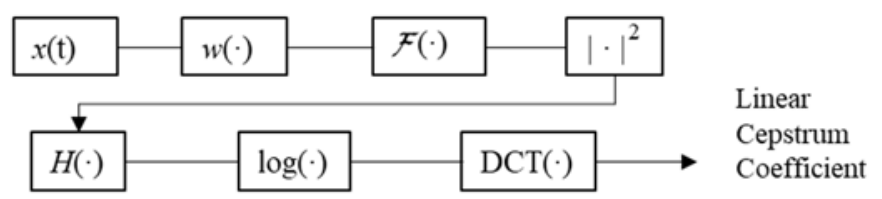

note: $w(\mathrm{t})$ : windowing $\mathcal{F}(\cdot)$ : FFT $H(\cdot)$ : Band-Pass Filter

Fig. 1. Block diagram of solving (a) power cepstrum and (b) linear cepstrum codfficient.

Compared with the power cepstrum solving process, the LCC has two more steps: windowing and linear band-pass filtering. LCC feature extraction consists of six steps: reshaping, windowing, fast Fourier transform, band-pass filter, logarithm, and discrete cosine 
transform (DCT). The block diagram of solving LCC is shown in Fig.1(b). After windowing, the AE signal is made stable and the frequency leakage can be avoided effectively. By using band-pass filter, the signal in the key frequency range can be analyzed according to the characteristics of AE signal. In order to ensure the stable output in the filter bank, the frequency band of adjacent filters is generally overlapped by half. The LCC uses the discrete cosine transform to replace the more complex inverse Fourier transform. The detailed steps for LCC calculating are as follows:

Step 1, reshaping. The AE signal is macroscopically non-stationary, and it can be regarded as a stationary signal only when the time window is very short. If the length of $\mathrm{AE}$ segment is too large, the power spectrum will be interfered by noise when using the Fourier transform. Therefore, it is necessary to divide the AE signal into small segments when solving the cepstrum coefficient. The first step in calculating LCC is to divide the AE signal into frames (i.e. segments of equal length), reshape each frame as a row, and convert the AE signal into a matrix, as shown in equation (1).

$$
x(t) \stackrel{\text { Reshaping }}{\longrightarrow}\left(\begin{array}{ccc}
x_{11} & \ldots & x_{1 N} \\
\vdots & \ddots & \vdots \\
x_{M 1} & \cdots & x_{M N}
\end{array}\right)
$$

where $x(t)$ represents the full waveform of AE signal, $N$ represents the number of AE data per frame length, $M$ represents the number of frames of AE signal.

Step 2, windowing. The second step is to do windowing for each frame which means multiply each row by a 'Hanning Window'. The purpose of windowing is to reduce the leakage in frequency domain and enhance the continuity of two adjacent frames. The frame signals after windowing are shown in equation (2).

$$
s[n]=w[n] x[n] \quad 1 \leq n \leq N
$$

where $x[n]$ represents a frame of AE signal, $w[n]$ represents the window function which is shown in equation (3).

$$
w[n]=(1-\alpha)-\alpha \cos \left(\frac{2 \pi n}{N-1}\right)
$$

where $\alpha$ represents the window coefficient, and for Hamming window $\alpha=0.46164$.

Step 3, fast Fourier Transform. The aim of this step is to get the spectrum of the signal.

Step 4, band-pass filter. In this step, the signal amplitude spectrum obtained in step 3 is multiplied by the linear filter bank to obtain the spectrum of the signal passing through different filters.

$$
W_{m}[k]=H_{m}[\mathrm{k}] *|F F T(s[n])|^{2}
$$

where $H_{m}[k]$ represents the transfer function of band-pass filter in linear filter bank, $F F T(s[n])$ represents the fast Fourier transform of signal $s[n]$.

$$
H_{m}(k)=\left\{\begin{array}{cc}
0 & (m-1) \cdot f_{s} \leq k<(m+1) \cdot f_{s} \\
1 & \text { others }
\end{array}\right.
$$


where, $f_{\mathrm{s}}$ represents the bandwidth of the band-pass filter, $m$ is the serial number of the filter in the filter bank.

Step 5, logarithm. Take the logarithm of the power spectrum output by the linear filter bank to get the logarithm power spectrum.

Step 6, discrete cosine transform (DCT). The LCC of each frame signal can be obtained by doing DCT for logarithm power spectrum. The LCC is obtained by equation (6).

$$
\mathbf{C}[u]=\sqrt{\frac{2}{M_{f}}} \sum_{m=1}^{M_{f}} \log 10\left(W_{m}[k]\right) \cos \left(\frac{\pi}{2 M_{f}}(2 m+1) u\right)
$$

where $C(u)$ is the $u$-th parameter of the LCC, $M_{\mathrm{f}}$ represents the number of filters in the linear filter bank.

\section{Experiment}

Limestone collected from Zhenchengdi Mine in Taiyuan, China is used for Brazilian split test. The raw limestone samples were collected underground, sealed with plastic film, transported to the laboratory in wooden boxes, and then processed into cylinders with a diameter of $50 \mathrm{~mm} \times 25 \mathrm{~mm}$.

The loading system used for the Brazilian test is the RMT-159 universal testing machine produced by Wuhan Geotechnical Institute of Chinese Academy of Sciences. The maximum load of the testing machine is $1000 \mathrm{kN}$, the deformation rate is $0.0001 \sim 1 \mathrm{~mm} / \mathrm{s}$, the range of force sensor is $1000 \mathrm{kN}$ with an accuracy of 3 \% F.S. The measuring range of axial displacement sensor is $5 \mathrm{~mm}$ with an accuracy of 1.5 \% F.S. Axial displacement control is used for the test, and the loading rate is set to be $0.010 \mathrm{~mm} / \mathrm{s}$. The sample frequency of load and displacement is set to by $10 \mathrm{~Hz}$.

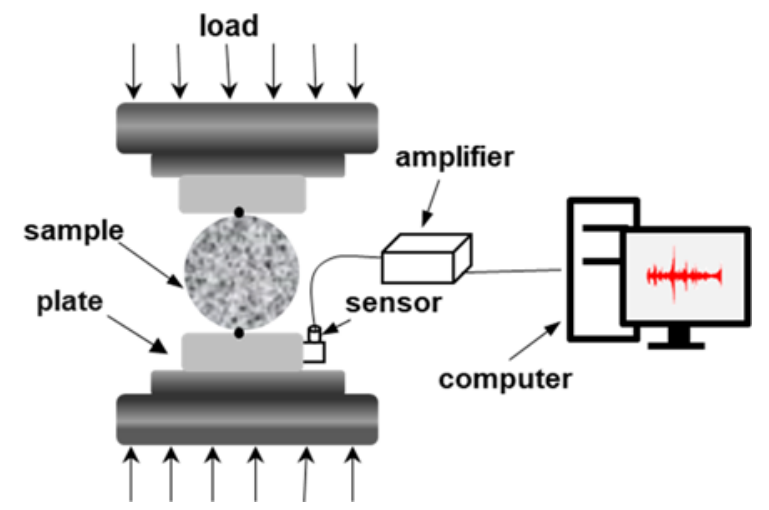

Fig. 2. Schematic diagram of experimental setup.

The AE acquisition instrument adopted the DS2 AE acquisition system produced by Beijing Softland Times Scientific \& Technology Co., Ltd., Beijing, China. The instrument enables continuous and real-time detection and acquisition of the full waveform of AE signal. The AE sensor RS2-A was used, at a frequency range from $50 \mathrm{kHz}$ to $400 \mathrm{kHz}$, a center frequency of $150 \mathrm{kHz}$, and an amplifier gain of $20 \mathrm{~dB}$. In this paper, the AE sensor was coated with petroleum jelly and attached to the pressure head of the test machine. The trigger mode of the $\mathrm{AE}$ acquisition instrument was set to manual trigger and the sampling frequency was set to $1 \mathrm{MHz}$. After the acquisition instrument had been triggered, the full waveform data of 
the $\mathrm{AE}$ was saved until the end of the experiment. A schematic diagram of the experimental device is shown in Figure 2.

\section{Results and Analysis}

Fig.3 shows the load-time curve of the limestone sample and the accompanying full waveform AE data. It can be seen from Fig. 3 that at the beginning of the experiment, there are few AE signals and the signal fluctuation is very small. With the increase of the load, the $\mathrm{AE}$ signal gradually increases, and the AE suddenly increases at the moment of sample failure. Before the failure of the limestone sample, the traditional AE parameters such as amplitude of AE signal, number of ring counts increase faster in the critical yield failure stage of rock samples, and thus AE can be used to monitor the failure of rock samples. But at the beginning of the loading, there is not much change in these AE parameters. Generally, AE signals in this early stage are rarely analyzed. However, when using LCC to analyze the AE signal in this stage, it demonstrates obvious change which is discussed in detail in the following section.
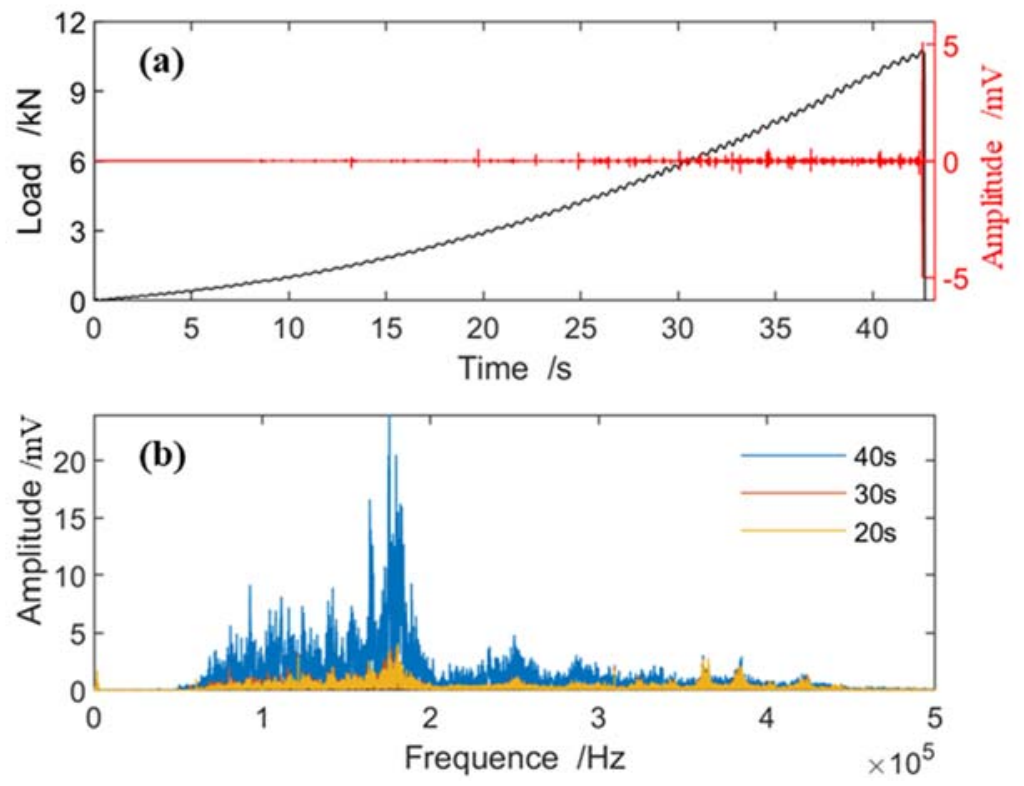

Fig. 3. Brazilian test results of limestone. (a) load vs time curve and accompanying full waveform of $\mathrm{AE}$ signal; (b) amplitude spectrum of AE at $20 \mathrm{~s}, 30 \mathrm{~s}$ and $40 \mathrm{~s}$.

It is calculated the LCC of AE signal by using every $0.1 \mathrm{~s} \mathrm{AE} \mathrm{segment} \mathrm{during} \mathrm{the} \mathrm{test.}$ Fig.4 shows the variation of LCC parameters. Each point in the figure represents the average value of LCC parameters within $0.1 \mathrm{~s}$, where LCC-1 to LCC-9 represent the 1st to 9th parameter of LCC respectively. It can be seen from Fig.4 that LCC-1 and LCC-3 increase along with the increase of the load, and the linear fitting correlation coefficients $\mathrm{R}^{2}$ are 0.472 and 0.581 , respectively. LCC- 2 and LCC-4 decrease with the increase of the load, and the linear fitting correlation coefficients $\mathrm{R}^{2}$ are 0.565 and 0.532 , respectively. Other parameters of LCC have poor correlation with the load. It can be seen that the first four parameters of LCC have strong correlation with the load in the process of rock failure under the test, but the values of LCC parameters fluctuate greatly. 

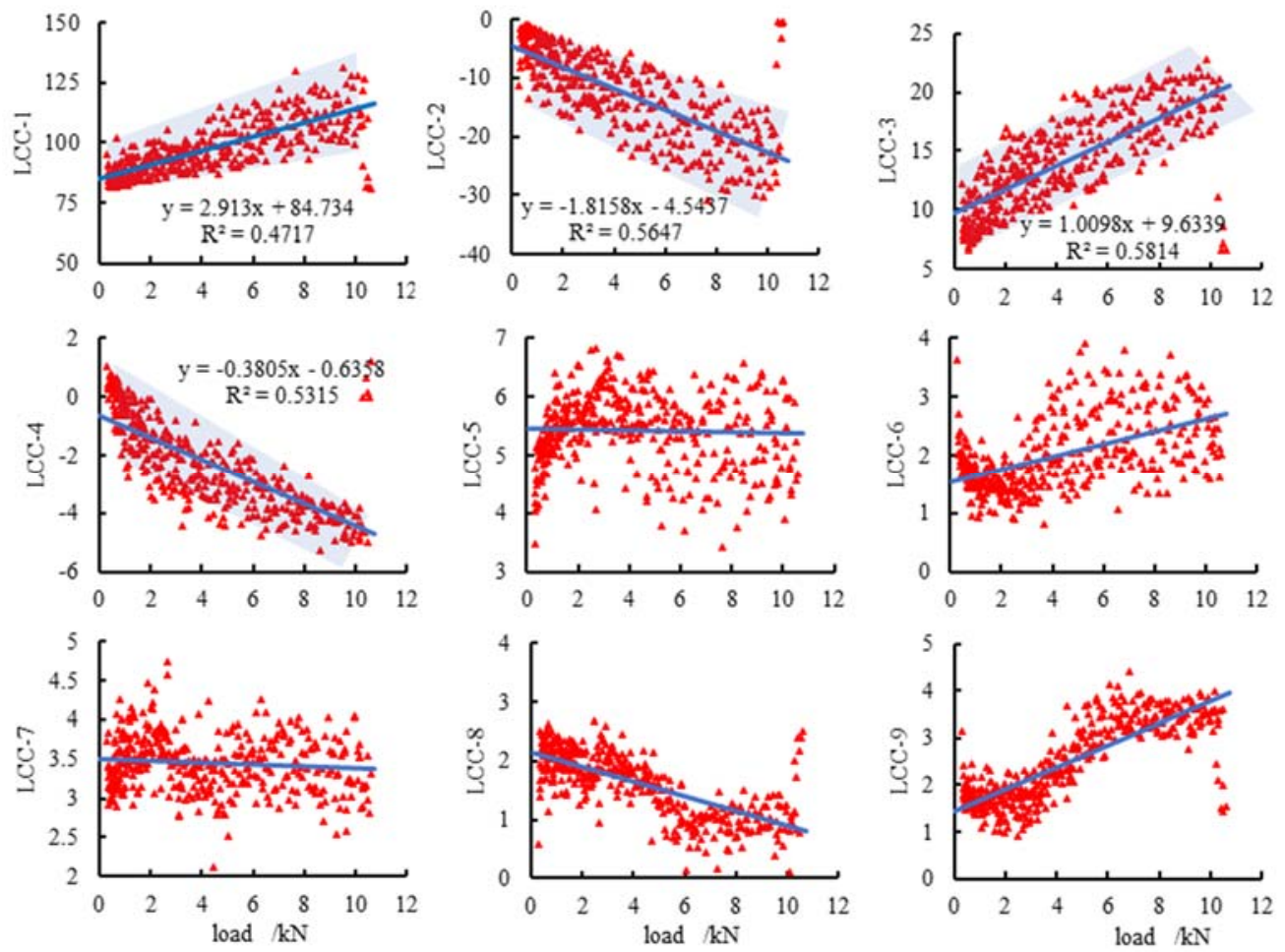

Fig. 4. Plot of LCC parameters of every $0.1 \mathrm{~s}$ AE signal segment with respect to load of limestone sample and their linear fitting.
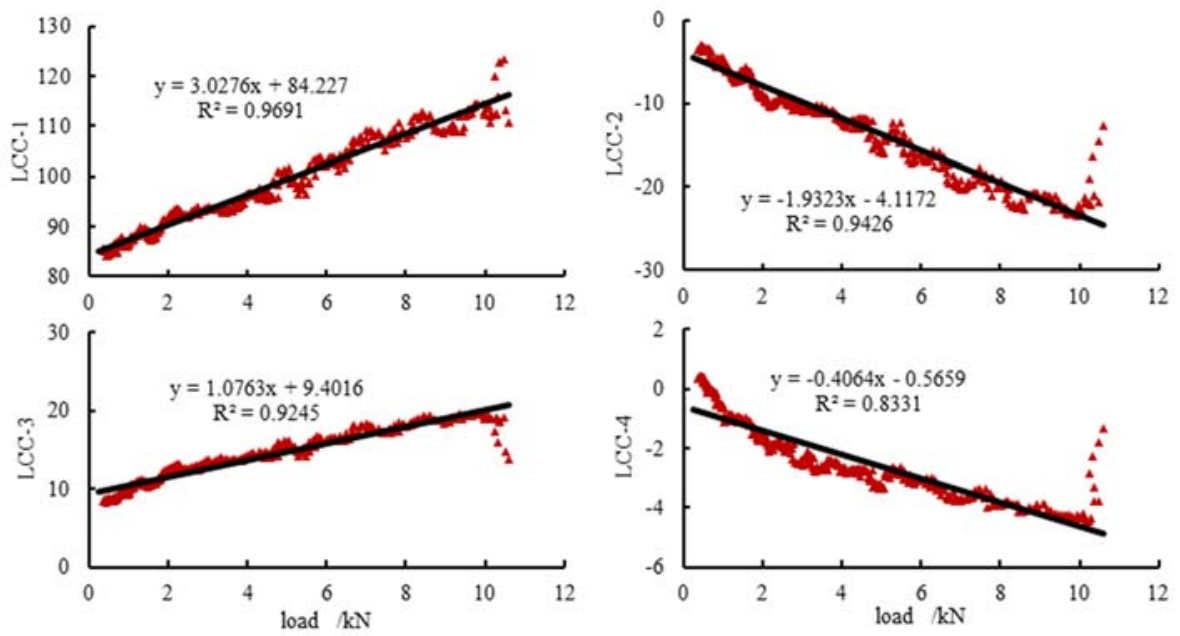

Fig. 5. Plot of LCC parameters of every $1 \mathrm{~s} \mathrm{AE} \mathrm{signal} \mathrm{segment} \mathrm{with} \mathrm{respect} \mathrm{to} \mathrm{load} \mathrm{of} \mathrm{limestone}$ sample and their linear fitting.

The mean of LCC of $1 \mathrm{~s}$ AE segment were obtained by doing 10-point averaging for LCCs in Fig.4, as shown in Fig.5. Fig.5 shows the variation of LCCs in every $1 \mathrm{~s}$ with tensile load and their linear fitting results. It can be seen that the linear fitting coefficients of LCC-1 to LCC-4 with the load are $0.969,0.943,0.925$ and 0.833 , respectively. In the process of sample failure, LCCs of AE show a high correlation with the load of sample. At the moment 
of sample failure, LCC-1 to LCC-4 show significant deviation from the linear fitting relationship, which can be regarded as the precursor of sample failure. It is worth noting that each point in Fig.5 is obtained by using every $1 \mathrm{~s} \mathrm{AE} \mathrm{segment,} \mathrm{which} \mathrm{is} \mathrm{independent} \mathrm{of} \mathrm{the}$ history and future trend of AE signals. This indicates that LCC of AE is capable to be a characteristic parameter for predicting tensile failure of rock. This characteristic parameter can hopefully be used to evaluate the stability of rock when combined with machine learning.

\section{Conclusion}

The Brazilian split test was carried out on limestone samples along with collecting full waveform AE signal during the entire loading process. The LCC of AE signal was extracted by using cepstrum analysis method and regarded as the characteristic parameter for predicting the tensile failure of rock samples. The correlation between the LCC of AE and the load of the samples was then analyzed. It was found that the LCC is an effective AE characteristic parameter. The first to fourth parameters of the LCC calculated using $1 \mathrm{~s} \mathrm{AE} \mathrm{waveform} \mathrm{meet}$ the linear relationship with the load of the samples, and the correlation coefficients are 0.969 , $0.943,0.925,0.833$, respectively. The LCC of AE is capable to be used as a characteristic parameter for predicting the tensile failure of rocks.

\section{Acknowledgements}

This work was financially supported by the National Natural Science Foundation of China (Nos. 51634001, 52011530037), and the Fundamental Research Funds for the Central Universities (No. FRFIP-20-01).

\section{References}

1. Z. Aliabadian, G. Zhao, A.R. Russell, Int. J. Rock Mech. Min. Sci., 119, 211-221 (2019)

2. F. Dai, S. Huang, K. Xia, Z. Tan, Rock Mech. Rock Eng., 43, 657-666 (2010)

3. S. Braun, Mech. Syst. Signal Pr., 128, 674-676 (2019)

4. J. Sandberg, M. Hansson-Sandsten, Signal Process, 92, 1290-1301 (2012)

5. R.B. Randall, J. Antoni, W.A. Smith, Mech. Syst. Signal Pr., 118, 716-741 (2019)

6. R.B. Randall, Mech. Syst. Signal Pr., 97, 3-19 (2017)

7. P. Borde, A. Varpe, R. Manza, P. Yannawar, Int. J. Speech Technol., 18, 167-175 (2015)

8. F.Z. Chelali, A. Djeradi, Int. J. Speech Technol., 20, 725-740 (2017)

9. B. Rajesh, D.G. Bhalke, Int. J. Speech Technol., 19, 551-563 (2016)

10. S.S. Tirumala, S.R. Shahamiri, A.S. Garhwal, R. Wang, Expert. Syst. Appl., 90, 250271 (2017)

11. J. Cao, T. Zhao, J. Wang, R. Wang, Y. Chen, Neurocomputing, 261, 231-241 (2017)

12. X.J. Liu, Study on rockburst characteristics of acoustic signal and dynamic warning method, Ph.D. Thesis: Guangxi University (2018)

13. Q. Mei, M. Gül, M. Boay, Mech. Syst. Signal Pr., 119, 523-546 (2019)

14. J.C. Hassab, R. Boucher, J. Sound Vib., 40, 321-335 (1975) 